\title{
EMERGING TRENDS IN ORAL DISPERSIBLE TABLET
}

\author{
Vishal N Patel*, M M Gupta ${ }^{1}$ \\ Jaipur College of Pharmacy, Jaipur (Rajasthan) India \\ *Corresponding Author's Email: Vishalpatel_2502@yahoo.com,Ph:08000140915
}

\begin{abstract}
:
Oral Dispersible tablets are well recognised dosage forms presented in the market. The various advantages that they offer to the patients in terms of massive revenues by line extension of products include a variety of advantages. The development of oral dispersible tablets has been formulated for pediatric, geriatric, and bed rest patients and for those people as well as patients who may not have access to water.several formulation provide and opportunity for product line extension especially for elderly persons will have difficulties in taking conventional oral dosage forms because of hand termors and dysphasia. Basically swallowing problems also are happen in young individuals because of their under developed muscular and nervous systems. In some cases such as motion sickness, coughing, and an unavailability of water,swallowing conventional tablets may be difficult or improper.

Key wordS: ODT, Mechanism of action, Method of preparation, Patented Technology, Super Disintegrants.
\end{abstract}

\section{INTRODUCTION:}

\section{CHARACTERISTICS OF ORAL DISPERSIBLE DELIVERY SYSTEMS ${ }^{1,2}$}

\section{EASY OF ADMINISTRATION:}

Oral Dispersible Delivery Systems are easy to administer and handle hence, leads to better patient compliance. Usually, elderly people experience difficulty in swallowing the conventional dosage forms (tablets, capsules, solutions and suspensions) because of tremors of extremities and dysphagia. Oral Dispersible tablets may offer a solution for these problems.

\section{TASTE OF THE MEDICAMENT:}

As most drugs are unpalatable, mouth dissolving delivery systems usually contain the medicament in taste masked form. Delivery systems dissolve or disintegrate in patient's mouth, thus releasing the active ingredients which come in contact with the taste buds and hence, taste masking of the drugs becomes critical to patient compliance.

\section{HYGROSCOPICITY:}

Several Oral Dispersible dosage forms are hygroscopic and cannot maintain physical integrity under normal condition from humidity which calls for specialized product packaging.

\section{FRIABILITY:}

In order to allow Oral Dispersible tablets to dissolve in the mouth, they are made of either very porous and soft- molded matrices or compressed into tablets with very low compression force, which makes the tablets friable and/or brittle which are difficult to handle, often requiring specialized peel-off blister packaging.

\section{MOUTH FEEL:}

Mouth feel is critical, and patients should receive a product that feels pleasant. Any large particles from the disintegrating tablet that are insoluble or slowly soluble in saliva would lead to an unpleasant gritty feeling. This can be overcome by keeping the majority of the particles below the detectable size limit. In some cases, certain flavours can imbibe an improved mouth feel perception, resulting in a product that is perceived as being less gritty, even if the only change is the flavour. Effervescence can be added to aid disintegration and improve mouth feel by reducing the "dryness" of a product.

\section{VARIOUS TECHNOLOGIES USED IN THE MANUFACTURE OF ODT:}

The performance of ODT depends on the technology used in their manufacture. The orally disintegrating property of the tablet is attributable to a quick ingress of water into the tablet matrix, which creates porous structure and results in rapid disintegration. Hence, the basic approaches to develop ODT include maximizing the porous structure of the tablet matrix, incorporating the appropriate disintegrating agent \& using highly water-soluble excipient in the formulation ${ }^{24}$. Following technologies have been used by various researchers to prepare ODT: -

a) Freeze-Drying or Lyophilization

b) Tablet Molding

c) Sublimation

d) Direct Compression

e) Cotton Candy Process

f) Mass-Extrusion

g) Wet granulation method

h) Spray Drying

\section{a) Freeze-Drying or Lyophilization ${ }^{26}$}

Freeze drying is the process in which water is sublimed from the product after it is frozen. This technique creates an amorphous porous structure that can dissolve rapidly.

A typical procedure involved in the manufacturing of ODT using this technique is mentioned here. The active drug is dissolved or dispersed in an aqueous solution of a carrier/polymer. The mixture is poured in the wells of the preformed blister packs. The trays holding the blister packs are passed through liquid nitrogen freezing tunnel to freeze the drug solution or dispersion. Then the frozen blister packs are placed in refrigerated cabinets to continue the freezedrying. After freeze-drying the aluminium foil backing is applied on a blister-sealing machine. Finally the blisters are packaged and shipped.

The freeze-drying technique has demonstrated improved absorption and increase in bioavailability. Remon and Corveleyn in one of their study found maltodextrins very useful in preparing oral dispersible tablets by lyophilization.

The major disadvantages of lyophilisation technique are that it is expensive and time consuming; Fragility makes conventional packaging unsuitable for these products and poor stability under stressed conditions. 


\section{b) TABLET MOLDING ${ }^{24,25}$}

Journal of Drug Delivery \& Therapeutics; 2013, 3(2), 199-206

The preparation of ODT using molding technology employs water-soluble ingredients so that the tablet dissolves completely and rapidly. The active ingredients in most cases are absorbed through the mucosal lining of the mouth. Molding process is of two type's i.e. solvent method and heat method. Solvent method involves moistening the powder blend with a hydro alcoholic solvent followed by compression at low pressures in molded plates to form a wetted mass (compression molding). The solvent is than removed by airdrying. The tablets manufactured in this manner are less compact than compressed tablets and posses a porous structure that hastens dissolution. The heat molding process involves preparation of a suspension that contains a drug, agar and sugar (e.g. Mannitol or lactose) and pouring the suspension in the blister packaging wells, solidifying the agar at the room temperature to form a jelly and drying at $30^{\circ}$ under vacuum. The mechanical strength of molded tablets is a matter of great concern. Binding agents, which increase the mechanical strength of the tablets, need to be incorporated. Taste masking is an added problem to this technology. To overcome this, Van Scoik incorporated taste masked drug particles. The taste masked drug particles were prepared by spray congealing a molten mixture of hydrogenated cottonseed oil, sodium carbonate, lecithin, polyethylene glycol, and an active ingredient into a lactose based tablet triturate form. Compared to the lyophilization technique, tablets produced by the molding technique are easier to scale up for industrial manufacture. Masaki uses an agar solution as a binding agent and a blister packaging as well as a mold to prepare an intrabuccally fast disintegrating tablet.

\section{c) SUBLIMATION ${ }^{20,21}$}

The key to rapid disintegration of ODT is preparation of a porous structure in the tablet matrix. To generate such a porous matrix, volatile ingredients are incorporated in the formulation, that is later subjected to a process of sublimation. Highly volatile ingredients like ammonium bicarbonate, ammonium carbonate, benzoic acid, camphor, naphthalene, urea, urethane and phthalic anhydride may be compressed along with other excipients into a tablet. This volatile material is then removed by sublimation leaving behind a highly porous matrix. Tablets manufactured by this technique have reported to usually disintegrate in 10-20 sec. Even solvents like cyclohexane, benzene can be used as pore forming agents.

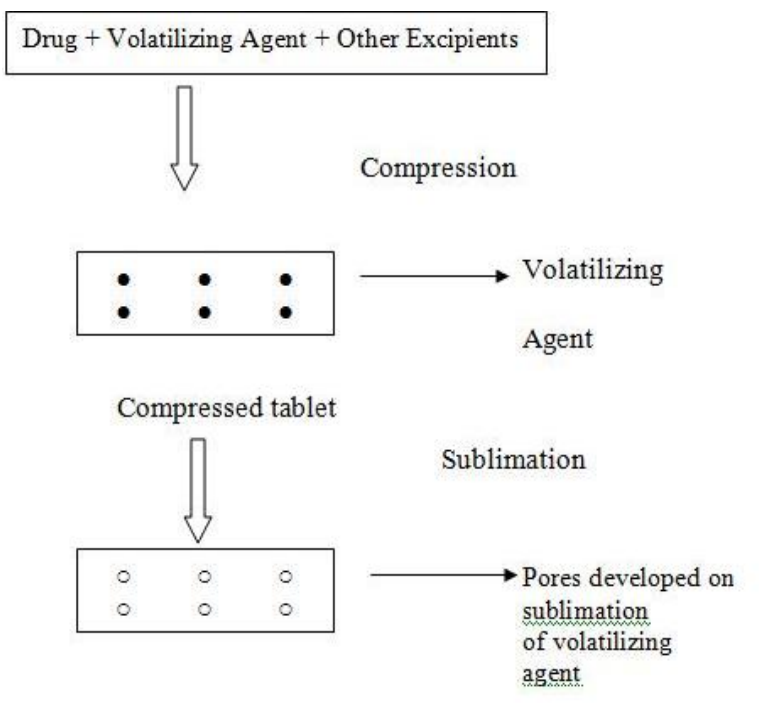

Figure 1: Sublimation method
Vacuum drying technique has been very often used by researchers to sublimate the volatile ingredients and thus maximize the porous structure in the tablet matrix. It is likely that a porous hydrophilic matrix will easily pick up the disintegrating medium and break quickly.

\section{d) DIRECT COMPRESSION ${ }^{24,25}$}

Direct compression represents the simplest and most cost effective tablet manufacturing technique. This technique can now be applied in the preparation of ODT because of the availability of improved excipients especially superdisintegrants \& sugar based excipients.

\section{(a) Superdisintegrants}

In many orally disintegrating tablet technologies based on direct compression, the addition of superdisintegrants principally affects the rate of disintegration and hence the dissolution. The presence of other formulation ingredients such as water-soluble excipients and effervescent agents further hastens the process of disintegration. Bi and Wantanbe have used microcrystalline cellulose (MCC) and low substituted hydroxyl propyl cellulose (HPC) to manufacture ODT. The ratio of MCC to HPC varied from 8:2 to 9:1. Ito and Sugihara investigated use of agar powder as a disintegrant because the powder absorbs water and swells without forming gel at physiological temperature. Ethylpharm (France) has introduced a Flash- dose technology, which contains coated crystals and micro granules along with the disintegrant. In this technology, two types of granules are used; a disintegrating agent (e.g. modified cellulose- croscarmellose) which has a high swelling force, and a swelling agent (e.g. starch) which has a low swelling force.

Shirwaikar and co-workers prepared Atenolol tablets by dry granulation method using three superdisintegrant, croscarmellose sodium (Ac-Di-Sol), crospovidone and sodium starch glycolate, and they found that Ac-Di-Sol was the best superdisintegrant among the three.

\section{(b) Sugar Based Excipients}

This is another approach to manufacture ODT by direct compression. The use of sugar based excipient especially bulking agents like dextrose, fructose, isomalt, lactilol, maltilol, maltose, Mannitol, sorbitol, starch hydrolysate, polydextrose and xylitol, which display high aqueous solubility and sweetness, and hence impart taste masking property and a pleasing mouth feel.

ODT formulators prefer to use a directly compressible Mannitol, which enables the preparation of robust tablets that can withstand processing and transportation. Specially textured directly compressible, spray-dried, or granulated Mannitol excipient has been designed to meet these needs. These excipients under defined manufacturing conditions gives a highly porous structure and friable exterior structure which helps in faster disintegration of ODT. This also provides a satisfactory mouth feel and is suitable for use in preparation of harder ODT by direct compression at low pressure.

\section{e) COTTON CANDY PROCESS ${ }^{19}$}

The cotton candy process is also known as the "candy floss" process and forms the basis of the technologies such as Flash Dose $^{\circledR}$ (Fuisz Technology). An ODT is formed using a candyfloss or shear form matrix; the matrix is formed from saccharides or polysaccharides processed into amorphous floss by a simultaneous action of flash melting and centrifugal force. The matrix is then cured or partially recrystallised to 
provide a compound with good flow properties and compressibility. The candyfloss can then be milled and blended with active ingredients and other excipient and subsequently compressed into ODT. However the high processing temperature limits the use of this technology to thermo stable compounds only.

\section{f) MASS-EXTRUSION ${ }^{19}$}

This technology involves softening the active blend using the solvent mixture of water-soluble polyethylene glycol and methanol and subsequent expulsion of softened mass through the extruder or syringe to get a cylinder of the product into even segments using heated blade to form tablets. The dried cylinder can also be used to coat granules for bitter drugs and thereby achieve taste masking.

\section{g) WET GRANULATION METHOD ${ }^{19}$}

This technology involves the binding of the mixture of drug and suitable filler with the binder, like starch paste or PVP. After binding of the mass, it is dried until little moisture remains in the mass. Then for production of the suitable size granules, the mass is passed through the sieve. After that the size reduction, superdisintegrant is added and other remaining materials, like talc or magnesium stearate are added and mixed. This mixture is than compressed to form tablets. Addition of the superdisintegrant can be done either during granulation, intra granulation or extra granulation. Sometimes superdisintegrant is added partly in the formulation i.e. $50 \%$ amount is added during granulation and remaining amount is added after granulation.

\section{h) SPRAY DRYING ${ }^{19-23}$}

Spray drying is used in pharmaceutical industries to produce highly porous powders. The processing solvent is evaporated rapidly by spray drying, which renders the product highly porous and thus can be used in manufacturing ODT.

In this technique, gelatine can be used as a supporting agent and as a matrix, Mannitol as a bulking agent and sodium starch glycolate or croscarmellose or crospovidone are used as superdisintegrant. Tablets manufactured from the spray-dried powder have been reported to disintegrate in less than 20 seconds in aqueous medium.

Allen and Wang have reported this technique for preparing fast dissolving tablets. The formulation contained bulking agent like Mannitol and lactose, a superdisintegrant like sodium starch glycolate \& croscarmellose sodium and acidic ingredient (citric acid) and/or alkaline ingredients (e.g. sodium bicarbonate). This spray-dried powder, which compressed into tablets showed rapid disintegration and enhanced dissolution.

\section{PATENTED TECHNOLOGIES FOR ORALLY DISINTEGRATINGTABLETS ${ }^{15-18}$}

1) Zydis Technology.

2) Durasolve Technology.

3) Orasolve Technology.

4) Flash Dose Technology.

5) Wow Tab Technology.

6) Flash Tab Technology.

7) Oraquick Technology.

8) Quick -Dis Technology.

9) Nanocrystal Technology.

\section{1) ZYDIS TECHNOLOGY:}

Zydis, the best known of the mouth-dissolving/disintegrating tablet preparations, was the first marketed new technology tablet. A Zydis tablet is produced by lyophilizing or freezedrying the drug in a matrix usually consisting of gelatine. The product is very lightweight and fragile, and must be dispensed in a special blister pack. The Zydis product is made to dissolve on the tongue in 2 to 3 seconds. A major claim of the Zydis product is increased bioavailability compared to traditional tablets. Because of its dispersion and dissolution in saliva while still in the oral cavity, there can be a substantial amount of pre-gastric absorption from this formulation. Any pre-gastric absorption avoids first-pass metabolism and can be an advantage to drugs that undergo a great deal of hepatic metabolism. There are some disadvantages to the Zydis technology. As mentioned earlier, the Zydis formulation is very lightweight and fragile, and therefore should not be stored in backpacks or the bottom of purses. Finally, the Zydis formulation has poor stability at higher temperatures and humidity. It readily absorbs water, and is very sensitive to degradation at humidity greater than $65 \%$.

\section{2) DURASOLV TECHNOLOGY:}

Durasolv is Cima's second-generation fastdissolving/disintegrating tablet formulation. DuraSolv has much higher mechanical strength than Orasolv due to the use of higher compaction pressures during tableting. DuraSolv product is thus produced in a faster and more cost-effective manner. DuraSolv is so durable that it can be packaged in either traditional blister packaging or vials. This technology is not compatible with larger doses of active ingredients, because the formulation is subjected to such high pressures on compaction. Unlike Orasolv, the structural integrity of any taste masking may be compromised with high drug doses. The drug powder coating in Durasolv may become fractured during compaction, exposing the bitter-tasting drug to a patient's taste buds. Therefore, Durasolv technology is best suited for formulations including relatively small doses of active compound. The tablets made by this technology consist of a drug, fillers and a lubricant and prepared by using conventional tableting equipment and have good rigidity. These can be packed into conventional packaging system like blisters. Due to higher force of compaction used, tablets prepared are rigid.

\section{3) ORASOLV TECHNOLOGY:}

Orasolv ${ }^{\circledR}$ is Cima's first orally disintegrating dosage form. It based on direct compression of an effervescent agent and taste masked drug. The use of effervescence causes a tablet to disintegrate rapidly in less than 1 min on contact with water or saliva leaving coated drug powder. This technique is frequently used to develop over the counter formulations. This technology can accommodate a wide range of active ingredient from $1 \mathrm{mg}$ to $500 \mathrm{mg}$. The effervescence occurs due to chemical reaction between organic acid such as citric acid, fumaric acid or maleic acid and a base such as sodium bicarbonate, potassium bicarbonate, bicarbonate, which result in generation of $\mathrm{CO} 2$. Effervescent disintegration agents evolve gas by means of chemical reaction called effervescent couple. Carbonates such as sodium bicarbonate, sodium carbonate, potassium bicarbonate and potassium carbonate, magnesium carbonate, and acids like citric, tartaric, fumaric, adipic and succinic are used. Microparticles, effervescent agents and other ingredient such as flavours, sweeteners, colorants and lubricants are blended and compressed at a low degree of compaction.

\section{4) FLASH:}

The FlashDose technology utilizes a unique spinning mechanism to produce a floss-like crystalline structure, much 
like cotton candy. This crystalline sugar can then incorporate the active drug and be compressed into a tablet. This procedure has been patented by Fuisz and is known as Shear form. The final product has a very high surface area for dissolution. It disperses and dissolves quickly once placed onto the tongue. Flash dose tablets consist of self-binding shear form matrix termed as "floss". Shear form matrices are prepared by flash heat processing and are of two types.

\section{5) WOWTAB TECHNOLOGY:}

The Wowtab mouth-dissolving/disintegrating tablet formulation has been in the Japanese market for a number of years. The WOW in Wowtab signifies the tablet is to be given "With out Water". The Wowtab technology utilizes sugar and sugar-like (e.g., mannitol) excipients. This process uses a combination of low mould ability saccharides (rapid dissolution) and high mould ability saccharide (good binding property).The two different types of saccharides are combined to obtain a tablet formulation with adequate hardness and mouth dissolution rate. Due to its significant hardness, the Wowtab formulation is a bit more stable to the environment than the Zydis or OraSolv. It is suitable for both conventional bottle and blister packaging. The Wowtab product dissolves quickly in 15 seconds or less.

\section{TECHNOLOGIES EMPLOYING HEATING PROCESS ${ }^{14}$}

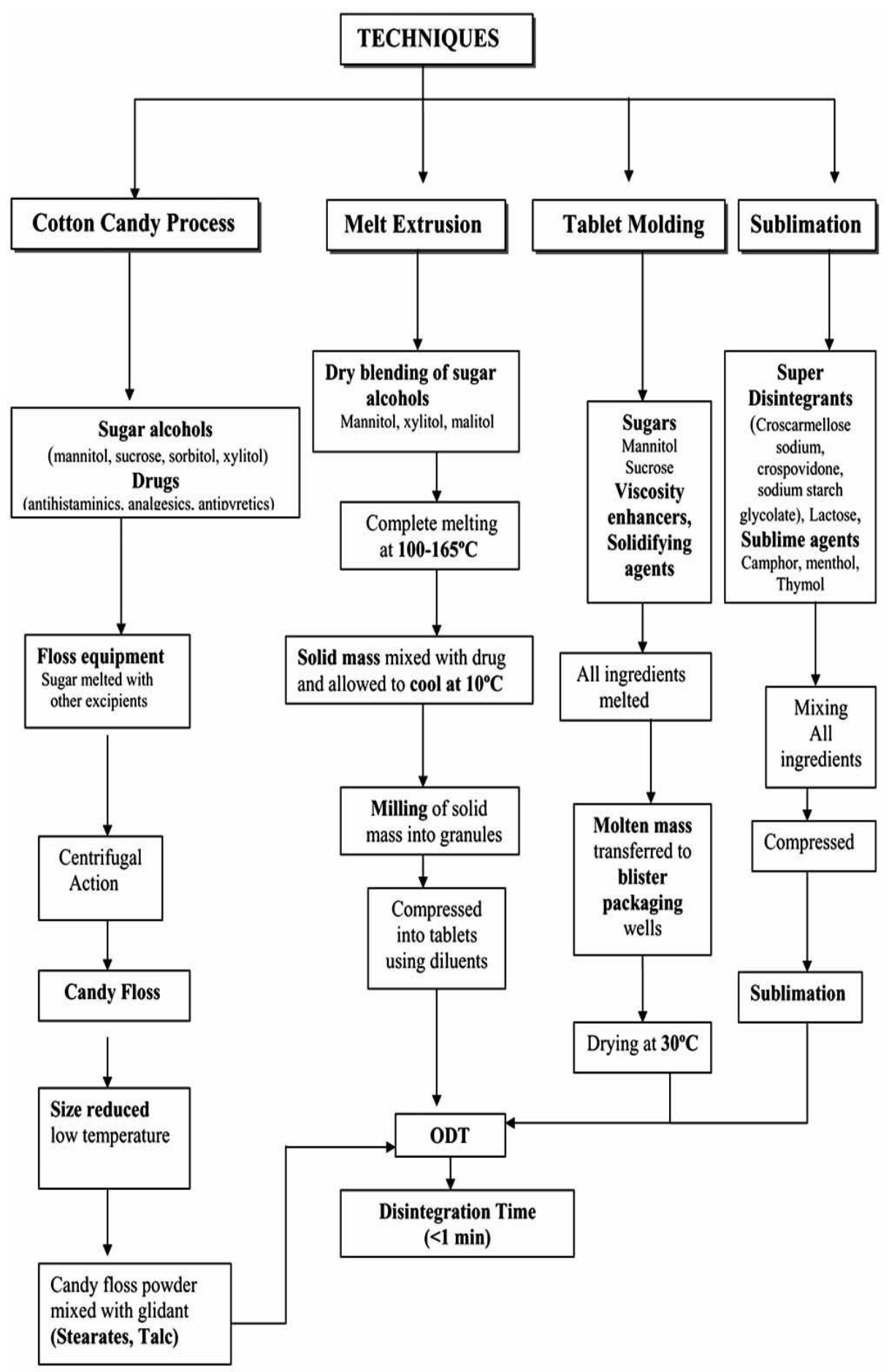

Figure 2: Schematic representation of the processes involved in the preparation of ODTs by employing heat based technology. 


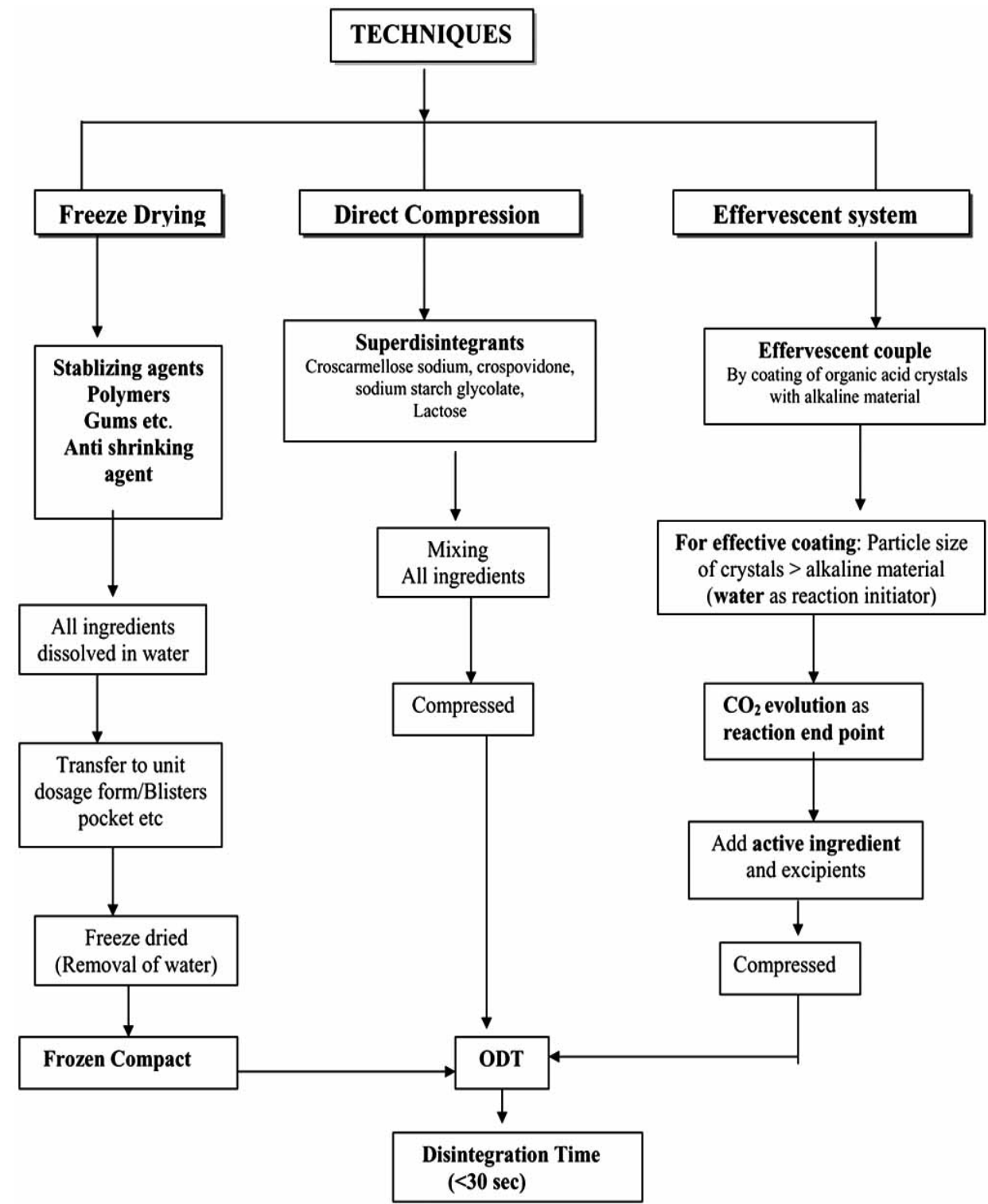

Figure 3: Schematic representation of the process involved in the different methods for preparation of ODTs without using heating process.

\section{6) FLASHTAB TECHNOLOGY:}

Prographarm laboratories have patented the Flashtab technology. This technology involves the preparation of rapidly disintegrating tablet which consists of an active ingredient in the form of microcrystal. Drug microgranules may be prepared by using the conventional techniques like coacervation, extrusion-spheronization, simple pan coating methods and microencapsulation. The microcrystal of microgranules of the active ingredient is added to the granulated mixture of excipients prepared by wet or dry granulation, and compressed into tablets. All the processing utilized the conventional tableting technology, and the tablets produced are reported to have good mechanical strength and disintegration time less than one minute.

\section{7) ORAQUICK TECHNOLOGY:}

OraQuick utilizes its own patented taste masking technology i.e. MicroMask®. In MicroMask ${ }^{\circledR}$ technology, taste masking process is done by incorporating drug into matrix microsphere. In this technique, tablet is prepared by dissolving the sugar (sucrose, mannitol, sorbitol, xylose, dextrose, fructose or mannose) and protein (albumin or gelatine) in a suitable solvent such as water, ethanol, isopropyl alcohol and ethanol-water mixture. The solution of matrix is then spray dried, yielding highly porous granules. Also, utilization of lower heat of production is advantageous for heat-sensitive drugs. Granules formed then mixed with drug and other excipients and compressed at low compression force. KV pharmaceuticals claimed that matrix formed protects and surrounds the drug powder in microencapsulated particles is more reliable during this step.

\section{8) QUICK -DIS TECHNOLOGY:}

Lavipharm has invented an ideal intra-oral mouth dissolving drug delivery system, which satisfies the unmet needs of the market. The novel intra-oral drug delivery system, trademarked Quick-Dis ${ }^{\mathrm{TM}}$, is Lavipharm ${ }^{\text {ee }}$ proprietary patented technology and is a thin, flexible, and quickdissolving film. The film is placed on the top or the floor of the tongue. It is retained at the site of application and rapidly releases the active agent for local and/or systemic absorption. The typical disintegration time is only 5 to 10 seconds for the Quick-Dis ${ }^{\mathrm{TM}}$ film with a thickness of $2 \mathrm{~mm}$.

\section{9) NANOCRYSTAL TECHNOLOGY:}

For orally disintegrating tablets, Elan's proprietary Nanocrystal technology can enable formulation and improve 
Vishal et al

Journal of Drug Delivery \& Therapeutics; 2013, 3(2), 199-206

compound activity and final product characteristics. Decreasing particle size increases the surface area, which leads to an increase in dissolution rate. This can be accomplished predictably and efficiently using Nanocrystal technology. Nanocrystal particles are small particles of drug substance, typically less than $1000 \mathrm{~nm}$ in diameter, which are produced by milling the drug substance using a proprietary wet milling technique. Nanocrystal colloidal dispersions of drug substance are combined with water-soluble ingredients, filled into blisters, and lyophilized. The resultant wafers are remarkably robust, yet dissolve in very small quantities of water in seconds.

\section{INTRODUCTION TO MOTION SICKNESS ${ }^{19-24}$ DEFNITION:}

Motion sickness is the uncomfortable dizziness, nausea, and vomiting that people experience when their sense of balance and equilibrium is disturbed by constant motion. Riding in a car, aboard a ship or boat, or riding on a swing all cause stimulation of the vestibular system and visual stimulation that often leads to discomfort.

\section{DESCRIPTION:}

Motion sickness is a common problem, with nearly 80 percent of the general population suffering from it at one time in their lives. People with migraine headaches or grave's syndrome, however, are more likely than others to have recurrent episodes of motion sickness. Researchers at the Naval Medical Center in San Diego, California, reported in 2003 that 70 percent of research subjects were suffering from severe motion sickness which had abnormalities of the vestibular system.

While motion sickness may occur at any age, it is more common in children over the age of two, with the majority outgrowing this susceptibility.

When looking at why motion sickness occurs, it is helpful to understand the role of the sensory organs. The sensory organs control a body's sense of balance by telling the brain what direction the body is pointing, the direction it is moving, and if it is standing still or turning. These messages are relayed by the inner ears (or labyrinth); the eyes; the skin pressure receptors, such as in those in the feet; the muscle and joint sensory receptors, which track what body parts are moving; and the central nervous system (the brain and spinal cord), which is responsible for processing all incoming sensory information.

Motion sickness and its symptoms surface when conflicting messages are sent to the central nervous system. An example of this is reading a book in the back seat of a moving car. The inner ears and skin receptors sense the motion, but the eyes register only the stationary pages of the book. This conflicting information may cause the usual motion sickness symptoms of dizziness, nausea and vomiting.

\section{CAUSES AND SYMPTOMS:}

While all five of the body's sensory organs contribute to motion sickness, excess stimulation to the vestibular system within the inner ear (the body's "balance center") has been shown to be one of the primary reasons for this condition. Balance problems, or vertigo, are caused by a conflict between what is seen and how the inner ear perceives it, leading to confusion in the brain. This confusion may result in higher heart rates, rapid breathing, nausea and sweating, along with dizziness and vomiting.
Pure optokinetic motion sickness is caused solely by visual stimuli, or what is seen. The optokinetic system is the reflex that allows the eyes to move when an object moves. Many people suffer when what they view is rotating or swaying, even if they are standing still.

Additional factors that may contribute to the occurrence of motion sickness include:

$>$ Poor ventilation.

$>$ Anxiety or fear. Both have been found to lower a person's threshold for experiencing motion sickness symptoms.

$>$ Heavy meal of spicy and greasy foods be avoided before and during a trip.

$>$ Alcohol. A drink is often thought to help calm the nerves, but in this case it could upset the stomach further. A hangover for the next morning's trip may also lead to motion sickness.

$>$ Genetic factors. Research suggests that some people inherit a predisposition to motion sickness. This predisposition is more marked in some ethnic groups than in others; one study published in 2002 found that persons of Chinese or Japanese ancestry are significantly more vulnerable to motion sickness than persons of British ancestry.

$>$ Pregnancy. Susceptibility in women to vomiting during pregnancy appears to be related to motion sickness, although the precise connections are not well understood.

\section{DIAGNOSIS:}

Most cases of motion sickness are mild and self-treatable disorders. If symptoms such as dizziness become chronic, a doctor may be able to help alleviate the discomfort by looking further into a patient's general health. Questions regarding medications, head injuries, recent infections, and other questions about the ear and neurological system will be asked. An examination of the ears, nose, and throat, as well as tests of nerve and balance function, may also be completed. Severe cases of motion sickness symptoms, and those that become progressively worse, may require additional, specific tests. Diagnosis in these situations deserves the attention and care of a doctor with specialized skills in diseases of the ear, nose, throat, equilibrium, and neurological system.

\section{TREATMENT OF MOTION SICKNESS:}

Most medications for motion sickness need to be taken at least 30 minutes before exposure to the activity that can cause the problem. Persons with glaucoma or prostate problems should not take most of these medications unless so advised by their doctor.

\section{Antihistamines}

$>$ Cinnarizine, Meclizine of the antihistamine family can cause drowsiness like other most other medications, it is best to take these before motion stimulation.

$>$ Dimenhydrinate (Dramamine). Similar to meclizine. Liquid forms are available.

$>$ Cyclizine is similar to meclizine. It is suitable for children 6 years of age or older as well as adults.

$>$ Haldol, Thorazine -- these anti-psychotic drugs have dopamine blocking activity which is useful for blocking nausea as well as stimulating stomach motion which helps clear food from the digestive tract.

$>$ Promethazine. This drug is one of the most effective available for motion sickness. One dose lasts up to 8 hours. Like the other drugs, it can cause drowsiness.

PHYSICO-MECHANICAL PARAMETERS Bulk Density ${ }^{11}$ 
Weighed quantity of Cinnarizine was transferred into $100 \mathrm{ml}$ measuring cylinder without tapping during transfer. The volume occupied by drug was measured. Bulk density was measured by using formula

$$
\begin{gathered}
\qquad \boldsymbol{\rho}_{\mathbf{i}}=\mathbf{m} / \mathbf{V}_{\mathbf{i}} \\
\text { Where, } \mathrm{m}=\text { mass of the blend } \\
\mathrm{V}_{\mathrm{i}}=\text { untapped volume }
\end{gathered}
$$

Tapped density ${ }^{12}$

Weighed quantity of drug was taken into a graduated cylinder. Volume occupied by the drug was noted down. Then the cylinder was subjected to $500,750 \& 1250$ taps in tap density apparatus.

$$
\begin{aligned}
\boldsymbol{\rho}_{\mathbf{t}} & =\mathbf{m} / \mathbf{V}_{\mathbf{t}} \\
\text { Where, } \mathrm{V}_{\mathrm{t}} & =\text { tapped volume } \\
\mathrm{m} & =\text { mass of the blend }
\end{aligned}
$$

\section{Carr's Index (Compressibility) ${ }^{13}$}

The compressibility index and Hausner ratio are measures of the property of powder to be compressed. The packing ability of drug was evaluated from change in volume, which is due to rearrangement of packing occurring during tapping. It was indicated as Carr's compressibility index was calculated as follows.

\section{Carr's index $=[$ Tapped density - Bulk density/Tapped density] X 100}

\section{Hausner Ratio ${ }^{13}$}

It is measurement of frictional resistance of the drug . The ideal range should be 1.2-1.5. It was determined by the ratio of tapped density and bulk density

\section{Hausner Ratio $=$ Tapped density $/$ Bulk density}

\section{Angle of Repose: ${ }^{14}$}

Weighed quantity of the drug was passed through a funnel kept at a height $2 \mathrm{~cm}$ from the base. The powder was passed till it forms a heap and touches the tip of the funnel. The radius was measured and angle of repose was calculated by using above formula. It is defined as the maximum angle that can be obtained between the free standing of powder heap and horizontal plane, which is given by the equation:

$$
\theta=\tan ^{-1} \mathrm{~h} / \mathrm{r}
$$

Where, $\theta=$ Angle of repose

$$
\begin{aligned}
\mathrm{h} & =\text { Height of powder heap, } \\
\mathrm{r} & =\text { Radius of the powder cone. }
\end{aligned}
$$

\section{SELECTION OF EXCIPIENTS:}

Following excipients were selected to fulfill the criteria of fast disintegrating tablet.

\section{(a) PVP k-30:}

In tableting, povidone solutions are used as binders in wet granulation processes. It was also aid in disintegration and dissolution. Povidone is used as a solubilizer in oral and parenteral formulations and has been shown to enhance dissolution of poorly soluble drugs from solid-dosage forms.

\section{(b) Microcrystalline Cellulose (Avicel 102):}

It is widely used as a diluent in tablets and capsules. It is used in both wet granulation and direct compression processes and also used as tablet disintegrant. It has both binding and disintegrant action.

\section{(c) Crosscarmellose Sodium:}

It swells 4-8 folds in 10 seconds. The cellulose derivative swells in two dimensions radially. In tablet formulations, croscarmellose sodium may be used in both direct compression and wet granulation processes. When used in wet granulation croscarmellose sodium is added to in both the wet and dry stages of the process so that wicking and swelling ability can both be utilized.

\section{(d) Sodium Starch Glycolate:}

Sodium starch glycolate is widely used in oral pharmaceuticals as a disintegrant in tablet and capsule formulations. It is commonly used in tablets prepared by either direct compression or wet granulation process. The usual concentration employed in a formulation is between 2$8 \%$. Disintegration occurs by rapid uptake of water followed by rapid and enormous swelling.

\section{(e) Sodium Bicarbonate $\left(\mathrm{NaHCO}_{3}\right)$ :}

Carbon dioxide generating agents like sodium hydrogen carbonate $\left(\mathrm{NaHCO}_{3}\right)$, it swells in the gastric fluid as it gets contact with the aqueous medium. Formation of $\mathrm{CO}_{2}$ and entrapment of that gas into the polymeric gel causes swelling of the dosage form resulting a bulk density less than 1 . It then remains buoyant and floats in the gastric fluid, resulting a prolonged gastric residence time

\section{(f) Mannitol:}

As a Diluent in tablets (10-90\% w/w). It is not hygroscopic and used with moisture sensitive active ingredients. Mannitol is commonly use as an excipient in the manufacture of chewable tablet and mouth dissolving formulation because of its negative heat of solution, sweetness and mouth feel.

\section{(g) Talc:}

Talc is also used as a lubricant in tablet formulations; in a novel powder coating for extended-release pellets; and as an adsorbant. However, it is widely used as a dissolution retardant in the development of controlled-release products.

\section{(h) Lactose:}

Lactose is widely used as filler or diluents in tablets and capsules, and to a more limited extent in lyophilized products and infant formula.

\section{(i) Magnesium stearate:}

Magnesium stearate is widely used in cosmetics, foods, and pharmaceutical formulations. It is primarily used as a lubricant in capsule and tablet manufacture at concentrations between $0.25 \%$ to $5.0 \% \mathrm{w} / \mathrm{w}$.

\section{DRUG EXCIPIENT INTERACTION STUDY}

The Drug -Excipients study was carried out by physical mixing and observations were recorded as a change in the colour.

\section{Study Methods:-}

\section{(a) Cinnarizine + Diluents (1:5) Ratio:-}

About $0.1 \mathrm{gm}$ of Cinnarizine $+0.5 \mathrm{gm}$ of Diluents were weighed accurately in a tarred Petri dish. Mixture was heated in oven at $30^{\circ}, 40^{\circ}, 50^{\circ}, 60^{\circ} \mathrm{C}$ different temperatures \& $75 \%$ relative humidity and color changed was observed at these temperatures. 
(b) Cinnarizine. + Binder (1:5) Ratio:-

About $0.1 \mathrm{gm}$ of Cinnarizine $+0.5 \mathrm{gm}$ Binder were weighed accurately in a tarred Petri dish and heated in oven at different temperature. Color changed was observed at these temperatures.

\section{(c) Cinnarizine + Lubricants (20:1) Ratio:-}

About 0.2 gm of Cinnarizine +0.01 gm of Lubricants were weighed accurately in a tarred Petri dish and heated in oven at

\section{REFERENCE:}

1. Yamamoto Y, Fujii M, Watanabe K, Tsukamoto M, Shibata Y, Kondoh M, Watanabe Y: Effect of powder characteristics on ora tablet disintegration. International Journal of Pharmaceutics 2009; 365: 116-120.

2. Abdelbary A, Elshafeey AH, Zidan G: Comparative effects of different cellulosic-based directly compressed orodispersable tablets on oral bioavailability of famotidine. Carbohydrate Polymers 2009; 77: 799-806.

3. Abdelbary G, Prinderre P, Eouani C, Joachim J, Reynier JP, Piccerelle P. The preparation of orally disintegrating tablets using a hydrophilic waxy binder. International Journal of Pharmaceutics 2004;278: 423-433.

4. Patel DM, Patel MM. Optimization of Fast Dissolving Etoricoxib Tablets Prepared by Sublimation Technique Indian J Pharm Sci, 70(1), 2008 Jan-Feb; 71-76.

5. Fini A, Bergamante V, Ceschel VC, Ronchi C, Alberto C, Moraes FD. Fast dispersible/slow releasing ibuprofen tablets. European Journal of Pharmaceutics and Biopharmaceutics 2008; 69: 335-341.

6. Yourong F, Jeong SH, Park K: Fast-melting tablets based on highly plastic granules. Journal of Controlled Release 2005; 109: 203-210.

7. Jeong SH, Park K. Development of sustained release fastdisintegrating tablets using various polymer-coated ion-exchange resin complexes. International Journal of Pharmaceutics 2008; 353: 195-204.

8. Yosra SR. Elnaggar, El-Massik MA, Abdallah OY, Ebian AER:Maltodextrin. A Novel Excipient Used in Sugar-Based Orally Disintegrating Tablets and Phase Transition Process AAPS Pharm Sci Tech. June 2010; 11(2): 25-29

9. Kalia A, khurana S, Bedi N. Formulation and evaluation of mouth dissolving tablets of oxcarbazepine. International Journal of Pharma Pharmaceutical Sciences Nov.-Dec. 2009; 1(1): 12-23

10. Subramanian S, Sankar V, Manakadan AA, Ismailand S, Andhuvan G. Formulation and evaluation of cetirizine dihydrochloride oro dispersible tablet Pak.J. Pharm .Sci. April 2010; 23(2): 232-235.

11. Furtado S, Deveswaran R, Bharath S, Basavaraj BV, Abraham S, Madhavan V. Development and characterization of orodispersible tablets of famotidine containing a subliming agent. Tropical Journal of Pharmaceutical Research December 2008; 7(4): 1185-1189.

12. Tekade NP, Bhajipale NS, Ganesan V, Thenge RR, Dewade DR, Formulation, and In Vitro Evaluation of Orodispersible Tablets of Lansoprazole. International Journal of Chem Tech Research Jan-Mar 2010; 2(1): 400-405. different temperature. Color changed was observed at these temperatures.

\section{(d) Cinnarizine + Superdisintegrants (1:1):-}

About $0.1 \mathrm{gm}$ of Cinnarizine $+0.1 \mathrm{gm}$ of Superdisintegrants were weighed accurately in a tarred Petri dish and heated in oven at different temperature. Color changed was observed at these temperatures.

13. Setty CM, Prasad DVK, Gupta VRM, Sa B. Development of Fast Dispersible Aceclofenac Tablets: Effect of Functionality of Superdisintegrants Indian J Pharm Sci 2008; Mar-Apr; 70(2): $180-185$.

14. Jeong SH, Park K. Development of sustained release fastdisintegrating tablets using various polymer-coated ion-exchange resin complexes. International Journal of Pharmaceutics 2008; 353: 195-204.

15. Indurwade NH, Rajyaguru TH, Nakhat PD. Novel Approach Fast Dissolving Tablets. Indian Drugs 2002; 39(8):405-409.

16. Hirani JJ, Rathod DA, Vadalia KR. Orally Disintegrating Tablets: A Review. Tropical Journal of Pharmaceutical Research April 2009; 8 (2): 161-167.

17. Kundu S, Sahoo PK. Recent Trends In The Developments of Orally Disintegrating Tablet Technology. Pharma Times April 2008; 40(4): 11-20

18. Bhowmik D, Chiranjib.B, Krishnakanth, Pankaj, Chandira RM. Journal of Chemical and Pharmaceutical Researc 2009; 1(1): 163-167.

19. Shukla D, Chakraborty S, Singh S, Mishra B Mouth Dissolving Tablets I: An Overview of Formulation Technology Sci Pharm 2009; 77: 309-326.

20. Prajapati BG and Ratnakar N. A Review on Recent patents on Fast Dissolving Drug Delivery System. International Journal of PharmTech Research July-Sept 2009; 1(3): 790-798.

21. Bharawaj S, Jain V, Sharma S, Jat RC, and Jain S. Orally Disintegrating Tablets: A Review. Drug Invention Today 2012;2(1):81-88.

22. Gavaskar B, Kumar SV, Sharan G, Nagaraju $m$ and Rao YM. Present investigations and future prospects of oral disintegrating tablets: a review. International journal of pharmaceutical sciences and research 2010; 1(8): 87-96

23. Gavaskar B, Kumar SV, Sharan G, Nagaraju m and Rao YM. Formulation aspects in the development of orodispersible tablets: an Overview. int j pharmacy and pharm sci June 2011; 2(3): 3842.

24. Kuchekar BS, Badhan AC, Mahajan HS. Formulation development of oraldispersible tablets of cinnarizine. Pharma Times 2003; 35:7-9.

25. Mehta K, Garala K, Basu, Bhalodia R, Joshi B, Charyulu RN. an emerging trend in oral drug delivery technology: Rapid disintegrating tablets. journal of pharmaceutical science and technology 2010; 2 (10): 318-329.

26. Remon JP, Corveleyn S. Freeze - Dried Disintegrating Tablets. US Patent 6,010,719;2000 